\title{
Licenciements collectifs et fermetures d'entreprise au Québec : le cas Wal-Mart
}

Collective Lay-offs and Plant Closures in Quebec : the Wall-Mars case

\section{Michel Coutu}

\section{(2) OpenEdition}

12 Journals

Édition électronique

URL : http://journals.openedition.org/travailemploi/3728

DOI : 10.4000/travailemploi.3728

ISSN : 1775-416X

Éditeur

DARES - Ministère du Travail

\section{Édition imprimée}

Date de publication : 15 mars 2007

Pagination : $39-50$

ISBN : ISSN : 0224-4365

ISSN : 0224-4365

\section{Référence électronique}

Michel Coutu, "Licenciements collectifs et fermetures d'entreprise au Québec : le cas Wal-Mart », Travail et Emploi [En ligne], 109 | janvier-mars 2007, mis en ligne le 15 mars 2009, consulté le 19 avril 2019. URL : http://journals.openedition.org/travailemploi/3728; DOI : 10.4000/travailemploi.3728 


\title{
Licenciements collectifs et fermetures d'entreprise au Québec: le cas Wal-Mart
}

\author{
Michel Coutu (*)
}

Deux questions parcourent cet article: celle de la spécificité du modèle social canadien, plus précisément québécois, face à celui existant aux États-Unis, tant sur le plan des relations sociales que du droit du travail; celle des limites du droit des licenciements collectifs. L'auteur présente le cadre juridique existant en la matière avec ses trois composantes (droit québécois, droit fédéral et accords collectifs) et, pour montrer ses insuffisances, développe un "cas limite», celui de Wal-Mart, entreprise transnationale de commerce de détail employant plus 1700000 salariés. La chaîne a en effet décidé en mai 2005 de fermer, en invoquant le manque de rentabilité, le seul établissement d'Amérique du Nord où un syndicat venait de réussir à s'implanter. Il y a de fortes présomptions pour que les deux événements soient liés. Les arguments avancés par les syndicats ont été rejetés par la Commission des relations du travail du Québec. Des requêtes en révision ont été déposée et, pour l'essentiel, rejetées. L'auteur élargit sa réflexion et souhaite, pour remédier au silence du droit québécois, l'adoption au Québec d'une législation inspirée en matière de licenciements collectifs de celle qui existe en Europe, avec une négociation portant non seulement sur les effets de la décision mais sur ses fondements.

Le droit du travail est l'objet d'une lutte incessante entre le droit individualiste d'essence libérale et le droit social produit à la fois de l'autonomie collective et de l'interventionnisme de l'État social(1). Dans cette lutte incessante et quels que furent les progrès du droit social, la position subordonnée de ce droit en regard des rapports juridiques de domination qui, en régime capitaliste, structurent la sphère économique, se révèle toujours au grand jour dans le cas décisif, celui où le fondement même du libéralisme économique - le droit de propriété (GurTVITCH, 1931) - est poussé dans ses derniers retranchements. Dans le droit québécois du travail, rien ne reflète mieux l'essence du libéralisme économique que les conflits qui portent sur les licenciements collectifs et les fermetures d'usine. Dans un contexte de globalisation où l'État semble au surplus décentré, où se fait jour une véritable crise de l'État social (voir HaBermas, 1981; RoSANVALLON, 1981; OfFE, 1984) et où le discours des partisans du marché et des transnationales apparaît hégémonique, il y a à la fois une exacerbation, sur le plan empirique, du phénomène des licenciements collectifs et un certain désarroi du droit social, lequel paraît impuissant, dans son état actuel, à endiguer ce phénomène.

(*) Professeur à l'école de relations industrielles de l'université de Montréal, email : michel.coutu@umontreal.ca

(1) Voir à ce sujet l'analyse classique de Hugo SinzHeImer (1976). Pour une vue d'ensemble, voir Ulrich ZACHERT (2006).

Encadré 1
Les étapes du conflit Wal-Mart
au Québec
Établie au Québec depuis 1994, Wal-Mart, «la
plus grande entreprise de détail au monde et le plus
grand employeur privé », y exploite 47 magasins et
emploie plus de 10000 salariés. Jusqu'à récem-
ment, aucun établissement de cette énorme trans-
nationale n'était syndiqué en Amérique du Nord.
En août 2004, le magasin Wal-Mart de
Jonquière au Québec devient le premier établis-
sement de la chaîne couvert par une accréditation
syndicale (voir plus loin), et ce dans toute l'Amé-
rique du Nord. En décembre 2004, les travailleurs
unis de l'alimentation et du commerce obtien-
nent une seconde accréditation, visant cette fois
l'ensemble des salariés d'un magasin Wal-Mart
à Saint-Hyacinthe, sans qu'aucune convention
collective ne soit intervenue à ce jour entre la multi-
nationale et ses salariés de Saint-Hyacinthe. À
Jonquière, plutôt que de négocier, Wal-Mart préfé-
rera fermer son magasin le 6 mai 2005 . Le 16 mai
2005, une accréditation partielle est accordée à
un syndicat représentant un groupe de salariés du
magasin Wal-Mart à Gatineau, accréditation dont
Wal-Mart tentera sans succès à aujourd'hui d'ob-
tenir la révocation.
Dans l'ensemble de ses établissements au
Québec, Wal-Mart mène une guérilla judiciaire
sans merci pour éviter la syndicalisation ou la
négociation collective des conditions de travail de
ses salariés.


Rien d'étonnant, dira-t-on, car tout cela se produit en contexte nord-américain, là où le libéralisme économique règne sans partage, où le syndicalisme est en déclin et où il n'existe pas de contre-pouvoirs efficaces, qu'ils soient politiques, économiques ou juridiques, pour s'opposer à la domination de la «pensée unique». Il faut cependant bien prendre en compte la complexité des phénomènes et voir que le Québec, contrairement au Canada anglais, luimême passablement différent du modèle américain, fait presque figure de forteresse du «droit social» en Amérique du Nord. Cet article présente les caractéristiques du Québec en matière de droit du travail et des licenciements collectifs (première partie) pour décrire ensuite un «cas limite» de fermeture d'entreprise, celle opérée par l'entreprise Wal-Mart Canada inc. à l'encontre de l'un de ses établissements québécois (deuxième partie).

\section{Le travail en contexte nord-américain et la spécificité du Québec}

Le Canada n'est pas les États-Unis, une réalité qui échappe souvent aux observateurs étrangers, tentés par l'amalgame. Et le Québec est aussi différent du reste du Canada que celui-ci peut l'être des États-Unis. Ces différences tiennent bien sûr à un ensemble de facteurs historiques, linguistiques, religieux, culturels et politiques que nous ne pouvons développer ici(2). Soulignons toutefois quelques différences centrales, par rapport au voisin américain mais aussi au Canada anglais, et ce uniquement dans le domaine du travail.

\section{Le niveau de syndicalisation}

Le Québec est en Amérique du Nord la région la plus fortement syndiquée, avec un taux de syndicalisation de $40 \%$. Ce taux tombe à moins de $30 \%$ au Canada anglais et bien plus bas encore aux ÉtatsUnis (11\%) (Rose, ChaIson, 2001). Il est certain que la procédure d'accréditation des syndicats joue un rôle important: alors que les lois états-uniennes du travail favorisent de longues campagnes électorales qui permettent à la direction de l'entreprise de peser de tout son poids (en recourant fréquemment à des pratiques illégales) pour contrer la syndicalisation, l'accréditation des syndicats au Québec se fait normalement par simple comptage des cartes d'adhésion, l'ordonnance d'un scrutin secret demeurant

(2) Pour une analyse classique, voir SEYMOUR M. LIPSET (1990). l'exception(3). À ce facteur, il faut ajouter l'impact des politiques publiques, beaucoup plus interventionnistes au Canada, et la moindre hostilité des employeurs à la syndicalisation (Rose, ChaIson, 1996).

\section{Le monopole syndical de représentation}

Celui-ci provient, comme c'est le cas partout en Amérique du Nord, du fameux Wagner Act de 1935 (4). Mais ce monopole a été progressivement renforcé, par rapport au modèle américain, par les initiatives ultérieures de l'État social en matière de rapports de travail: par exemple, la retenue syndicale obligatoire est imposée par le Code du travail du Québec(5), tout comme l'arbitrage obligatoire des griefs (6), dont le processus est en partie contrôlé par le syndicat. Le monopole de représentation, tel qu'on le connaît en droit québécois et canadien, concentre un pouvoir économique, politique et juridique considérable entre les mains des organisations syndicales.

\section{L'exercice du droit de grève}

Comme partout en Amérique du Nord, l'exercice du droit de grève est fortement réglementé: interdiction de la grève en cours de convention collective(7), respect des services essentiels dans le secteur public, adoptions fréquentes de sévères lois de retour au travail dans les services publics. Cela dit, l'efficacité de la grève comme moyen d'action syndicale n'est pas remise en question par les syndicats québécois, qui peuvent compter - fait unique en Amérique du Nord - sur une interdiction légale $\mathrm{du}$ recours aux briseurs de grève(8). Et contrairement à la situation qui prévaut aux États-Unis, le droit de grève est reconnu aux travailleurs du secteur public (9).

\section{La structure de la négociation collective et le champ d'application de la négociation}

En Amérique du Nord, l'unité de négociation est normalement l'établissement (sauf dans l'industrie de la construction) et la négociation collective demeure fortement décentralisée. Toutefois, ce qui est tout à fait particulier, le Québec connaît un régime centralisé de négociation dans le secteur

(3) Voir Weiler (1983); Johnson (2004). C'est la loi Taft-Hartly de 1947 qui a reconnu le droit de l'employeur à la «liberté d'expression» aux États-Unis, ce qui a rapidement perverti tout le processus d'accréditation. Sur ce point voir ADAMS (1995).

(4) National Labor Relations Act, 29 U.S.C. $\S \S 151-169$.

(5) Code du travail, L.R.Q., c. C-27, art. 47.

(6) C. t., art. 100.

(7) Art. 107 C.t.

(8) Art. 109.1 C.t.

(9) Le degré d'organisation et la puissance du syndicalisme dans le secteur public canadien et québécois expliquent aussi le maintien d'un niveau de syndicalisation beaucoup plus élevé qu'aux États-Unis. Voir Clarkson (2002). 
public (10), lequel concerne environ un demi-million de salariés. Par ailleurs, le Québec, tout comme le reste du Canada d'ailleurs, ignore la distinction entre les matières obligatoires et facultatives de négociation introduite par la Cour suprême des États-Unis dans l'affaire Borg-Warner(11). Cette distinction permet à un employeur de se soustraire à une plainte pour refus de négocier de bonne foi si l'objet de la négociation porte sur des questions de «gestion interne », telles la fermeture ou le déménagement de l'entreprise, ou la sous-traitance. Au Canada, tout ce qui peut faire l'objet de la négociation collective est considéré comme constituant une condition de travail(12).

\section{Le pluralisme syndical}

Alors que les États-Unis, depuis la fusion de 1'American Federation of Labour (AFL) avec le Congress of Industrial Organizations (CIO), n'ont connu pour l'essentiel, tout comme le Canada anglais avec le Congrès du travail du Canada (CTC), qu'un régime syndical moniste (traversé toutefois par de nombreuses luttes d'influence entre puissantes fédérations syndicales telles les Teamsters, les Machinistes, les Travailleurs unis de l'auto, etc.(13) ), le Québec a fait l'expérience, pour des raisons à la fois religieuses, idéologiques et linguistiques, d'une différenciation du mouvement syndical en plusieurs pôles, grands et petits. L'existence de ce pluralisme syndical a permis aux conflits de valeurs de s'exprimer ouvertement, plutôt qu'en empruntant la forme indirecte de luttes territoriales de pouvoirs, à l'américaine, entre fédérations syndicales. Le fait que les travailleurs ont régulièrement l'occasion de réaffirmer ou de modifier leur affiliation syndicale a favorisé le maintien d'un niveau généralement élevé de démocratie syndicale et une identification plus immédiate de la «base», certes à des degrés éminemment variables, avec les directions syndicales.

\section{Le poids politique et social des organisations syndicales}

À plus d'une reprise, les syndicats québécois ont démontré leur capacité à ébranler sérieusement le pouvoir politique (par exemple lors du «Front commun» de 1972 ou des grèves du secteur public en 1983). Le mouvement syndical nord-américain a une tradition historique de luttes dures, mais cette tradition, en règle générale, s'est effritée depuis quelques décennies, au point de disparaître à peu

(10) Loi sur le régime de négociation des conventions collectives dans les secteurs public et parapublic, L.R.Q., c. R-8.2.

(11) Labor Board v. Borg-Warner Corp, 356 U.S. 342 (1958).

(12) Syndicat catholique des employés de magasin de Québec c. Cie Paquet Ltée, [1959] R.C.S. 206. Voir infra, conclusion.

(13) Ce qui a conduit récemment (juillet 2005) au départ de l'AFL-CIO des Teamsters et de la Fédération internationale des employés de service. près complètement aux États-Unis (14). C'est moins le cas au Canada(15) et certainement pas le cas au Québec. Par exemple, une grève politique d'une journée au Québec en novembre 2003, avec des actions spectaculaires comme le blocage des ports et de routes régionales, a suffisamment marqué les esprits pour que le gouvernement du Parti libéral renonce, en partie du moins, à son programme de contre-réformes et de démantèlement de l'État social. En l'absence d'un grand parti socialiste de gauche (qui n'a jamais vu le jour à cause de l'absence de solution à la «question nationale»)(16), ce sont les grandes centrales syndicales (Fédération des travailleurs du Québec, Confédération des syndicats nationaux, Centrale syndicale du Québec) qui jouent le rôle d'opposition, sur le plan social, aux gouvernants successifs.

Il faut toutefois insister sur une autre singularité, négative celle-là : l'institution au Québec, de 1969 à 2002, d'un système très particulier de mise en œuvre du droit des rapports collectifs de travail (ce qu'on appelle abusivement le «Code du travail» du Québec). Plutôt que de s'en remettre à un organisme de droit public chargé de la régulation juridique des rapports collectifs de travail (comme c'est le cas au Canada anglais avec les commissions des relations de travail - Labour Relations Boards - rendant des décisions de manière rapide, souple et non formaliste sur tous les aspects du droit des rapports collectifs), pendant toute cette période le Québec décida de faire appel à un modèle bien davantage judiciarisé et n'exerçant qu'une compétence civile restreinte: c'est ce qu'on appela le système à double paliers, avec les «commissaires du travail» et le «tribunal du travail»(17). Ce système éclaté, parcellisé et inutilement complexe (au surplus, manquant de

(14) Alors que les syndicats canadiens ont dans l'ensemble bien résisté aux demandes patronales visant à imposer des reculs dans les conditions de travail, la situation est différente aux USA, où les syndicats ont été acculés à la défensive et ont dû accepter de lourdes concessions. Voir Rose, CHAison (2001) p. 37.

(15) On tiendra compte également, par comparaison avec les États-Unis, de l'existence d'un parti social-démocrate influent, lié institutionnellement aux syndicats, au Canada anglais: le nouveau parti démocratique (NPD). En dépit de résultats électoraux fluctuants et de son incapacité à s'élever au-delà du statut de tiers-parti sur la scène politique fédérale, le NPD s'est souvent révélé apte à influencer de manière progressiste l'orientation des politiques publiques en matière de travail. Voir ADAMS, op. cit.

(16) Soulignons toutefois l'émergence, à la gauche du Parti québécois (parti indépendantiste, qui se dit parfois «socialdémocrate»), d'une organisation socialiste, "Québec Solidaire».

(17) Le tribunal du travail était formé de juges de la Cour du Québec et siégeait pour l'essentiel en appel des décisions des commissaires du travail, qui eux étaient des fonctionnaires du ministère du Travail. Or les commissaires n'avaient juridiction que sur certains aspects bien délimités du Code du travail (par exemple : les congédiements pour activités syndicales, mais non l'intimidation ou les menaces à l'endroit des militants syndicaux). Dans les autres cas, seule une plainte pénale était possible. 
cohérence vu l'individualisme des décideurs) n'a pu produire une rationalisation globale du droit des rapports collectifs de travail. Jusqu'à son abolition en 2002 et son remplacement par un tribunal administratif unique (la nouvelle Commission des relations du travail), ce système à double paliers a entraîné une judiciarisation excessive, à l'avantage surtout des avocats patronaux et des employeurs (un peu à l'instar du système américain, qui se caractérise également par un niveau élevé de judiciarisation) (ADAMS, op. cit.).

Mis à part ce dernier élément et en dépit des caractéristiques précédemment mentionnées favorisant le droit social, il ne faudrait pas concevoir le Québec comme une sorte de village gaulois, luttant «encore et toujours» (et surtout, «avec succès») contre l'envahisseur néo-libéral et globalisant. En fait, la «potion magique» qui a permis au droit social de maintenir sa vitalité s'appuyait, au-delà de l'autonomie collective (syndicale, mais aussi communautaire et populaire) en grande partie sur l'appareil de l'État qui lui était favorable (en dépit des reculs ponctuels, à l'effectivité limitée, imposés par les gouvernants). Cette alliance stratégique entre de larges pans de l'appareil administratif de l'État et le mouvement syndical et communautaire a survécu jusqu'à maintenant.

Dans la mesure toutefois où l'État social québécois apparaît lui-même en crise, une crise sur laquelle l'État n'a qu'une emprise somme toute limitée puisqu'elle relève en partie de la dynamique de la mondialisation(18), le droit social peine à retrouver sa vigueur et à faire face efficacement aux problèmes nouveaux qui surgissent, en particulier celui des fermetures d'usine et des licenciements collectifs, lequel prend les proportions d'une véritable épidémie.

Dans ce qui suit, nous allons brièvement présenter le droit applicable en matière de licenciements collectifs au Québec aux niveaux provincial et fédéral; et discuter plus longuement d'un aspect de la question, les fermetures motivées par l'antisyndicalisme de certaines entreprises, ce qui est le cas de l'entreprise Wal-Mart au Québec. En conclusion, nous mettrons l'accent sur les lacunes du droit québécois en la matière et tenterons d'identifier certaines voies de solution.
Le cadre juridique:

la faiblesse persistante du droit social en matière de licenciements collectifs

À la différence du droit français, le droit en vigueur au Québec en matière de licenciements collectifs demeure extrêmement laconique: ceci vaut tant pour les normes législatives elles-mêmes que pour la parcimonie des commentaires doctrinaux ou des décisions jurisprudentielles. Toutefois, vu l'importance des dispositions en la matière contenues fréquemment dans les conventions collectives, il faut départager le droit étatique, applicable à tous les salariés (syndiqués ou non), et le droit issu de l'autonomie collective, c'est-à-dire le droit des rapports collectifs de travail.

\section{Le droit étatique}

Il nous faut en outre distinguer le droit provincial, lequel concerne $90 \%$ des salariés québécois du droit fédéral, présent toutefois dans des secteurs-clé de l'économie.

\section{Le droit québécois (provincial) du travail}

Depuis 2002(19), les dispositions relatives au licenciement collectif font l'objet des articles 84.0.1. à 84.0.15 de la loi sur les normes du travail (ci-après la «L. n. t.»)(20). Auparavant, les dispositions pertinentes se trouvaient, depuis 1969, dans la Loi sur la formation et la qualification professionnelles de la main-d'œuvre (BÉLIVEAU, 2003). Ces dispositions ne donnaient ouverture qu'à un recours pénal et en fait n'étaient guère respectées par les employeurs, qui en contournaient les normes souvent en toute impunité. Le principal changement depuis le $1^{\text {er }}$ mai 2003 est l'existence d'une indemnité compensatrice au bénéfice des salariés.

L'article 84.0.1. L. n. t. définit le licenciement collectif comme étant une cessation du travail du fait de l'employeur «qui touche au moins dix salariés d'un même établissement au cours d'une période de deux mois consécutifs ». Avant de procéder à un tel licenciement pour des raisons économiques ou technologiques, l'employeur doit fournir un avis

(19) L.Q. 2002, c. 89, a. 49

(20) L.R.Q., c. N-1.1. La loi sur les normes du travail établit des normes minimales (salaire, heures de travail, vacances, congés, état de grossesse, interdiction du congédiement sans cause juste et suffisante, etc.) à l'intention des salariés non syndiqués. Mais certaines dispositions qui y sont prévues complètent le Code du travail et ne concernent que les salariés régis par une convention collective (voir les articles 87.1. et s., relatifs aux clauses de «disparité de traitement», c'est-à-dire discriminatoires à l'endroit des salariés ayant le moins d'ancienneté). D'autres dispositions concernent tant le secteur syndiqué que non syndiqué : par exemple, celles relatives au harcèlement psychologique ou aux licenciements collectifs. 
au ministre responsable; le délai de production de l'avis varie en fonction du nombre de salariés visés par le licenciement (21). Le ministre demande alors la constitution d'un comité d'aide au reclassement (art. 84.0.9) : ce comité est formé de l'employeur et du syndicat, ou, s'il s'agit d'un milieu non syndiqué, de représentants choisis par les salariés visés par le licenciement, chaque partie disposant d'un seul vote. Le comité paritaire a pour mission de fournir l'aide voulue aux salariés visés pour minimiser l'impact des licenciements et favoriser le maintien ou la réintégration en emploi de ces salariés. Plus précisément, le comité est chargé de l'élaboration d'un plan de reclassement «visant le maintien ou la réintégration en emploi de ces salariés et de veiller à la mise en ouvre de ce plan »(22). Les coûts de fonctionnement du comité d'aide au reclassement sont assumés par l'employeur, après entente avec le ministre ou conformément aux montants déterminés par le gouvernement (art. 84.0.11 L.n.t.). S'il y a défaut de donner l'avis de la part de l'employeur ou avis insuffisant, celui-ci doit verser à chaque salarié une indemnité (de l'ordre du salaire habituel) correspondant au délai d'avis auquel il est tenu(23). Cette possibilité, tel que mentionné, est de droit nouveau: la seule sanction possible pour l'employeur pris en défaut de transmission de l'avis de licenciement demeurait auparavant une sanction pénale, rarement sinon jamais appliquée (24).

\section{Le droit fédéral}

Le droit fédéral s'applique aux rapports du travail lorsque l'entreprise relève de la compétence constitu-

(21) Le délai est de deux, trois ou quatre mois, suivant qu'il y a moins de 100 , de 100 à 300 , ou 300 salariés et plus visés par le licenciement.

(22) Art. 84.0.10 L. n. t.

(23) Art. 84.0.13. L. n. t. Soulignons qu'en vertu de l'article 82 L. n. t. l'employeur est également tenu de donner un avis individuel au salarié qu'il licencie ou met à pied pour six mois ou plus : la durée de cet avis varie d'une semaine «si le salarié justifie de moins d'un an de service continu, de deux semaines s'il justifie d'un an à cinq ans de service continu, de quatre semaines s'il justifie de cinq à dix ans de service continu et de huit semaines s'il justifie de dix ans ou plus de service continu». Le salarié ne peut cumuler les indemnités prévues aux articles 83 et 84.0.13 LNT, mais a droit à l'indemnité la plus élevée. (24) Voir Albert c. Abitibi-Consolidated inc., [2003] IIJCan 33118 (C. Q.). Dans cette décision, la Cour du Québec rejette une réclamation en dommages-intérêts présentée par 414 salariés licenciés par Abiti-Consolitaded inc. La Cour «est d'accord avec l'argument de la défenderesse à l'effet que le seul droit dont les employés bénéficiaient était celui relié à la constitution d'un comité de reclassement pour les aider à retrouver un emploi... Par conséquent, ils ne peuvent réclamer le salaire qu'ils estiment avoir perdu et la seule sanction imposable à l'employeur pourrait être une amende. Il est évident que l'analyse de la situation serait différente si on devait appliquer la législation en vigueur depuis le 1er mai 2003. En effet, les règles relatives à l'avis de licenciement collectif sont maintenant insérées dans la loi sur les normes du travail et on y prévoit maintenant une indemnité compensatrice à défaut d'un préavis de licenciement collectif suffisant». tionnelle fédérale (25), ce qui concerne environ $10 \%$ de la main-d'œuvre au Québec. Le Code canadien du travail(26) (ci-après C. c. t) prévoit, à l'instar de la législation québécoise, l'obligation de donner un avis de licenciement collectif lorsque cinquante employés ou plus sont visés et ce, que les licenciements surviennent de manière simultanée ou échelonnée sur au plus quatre semaines. L'avis doit être produit au moins quatre mois avant la date du premier licenciement prévu(27). L'avis est donné au ministre fédéral du Travail, au ministre fédéral du Développement des ressources humaines, à la Commission de l'assuranceemploi du Canada, à tous les syndicats concernés ou, à défaut, aux surnuméraires eux-mêmes. L'employeur doit coopérer avec la Commission afin de faciliter le réemploi des travailleurs $(28)$.

De manière similaire à ce qu'exige la législation québécoise du travail, le Code canadien du travail prévoit la mise sur pied d'un «comité mixte de planification»: toutefois, c'est ici l'employeur qui est responsable de la constitution de ce comité. Il s'agit d'un comité paritaire composé d'au moins quatre membres, représentant pour moitié l'employeur, pour moitié les syndicats concernés: à défaut de syndicat, les surnuméraires désignent leurs représentants (29). Le comité de planification doit se réunir dans les deux semaines de l'avis de licenciement collectif. Il a pour mission d'élaborer un programme d'adaptation visant soit à éliminer la nécessité des licenciements, soit à «minimiser les conséquences de cette mesure pour les surnuméraires et les aider à trouver un autre travail» (30). Remarquons par ailleurs qu'un inspecteur du travail peut surveiller la constitution et le fonctionnement du comité, et assister aux réunions à titre d'observateur.

Après un délai de six semaines suivant la remise de l'avis de licenciement collectif, l'une ou l'autre des parties - pourvu que tous les membres qui la constituent soient d'accord-peut demander au ministre fédéral du Travail de nommer un arbitre, si le comité «n'a pas encore élaboré un programme d'adaptation » ou si ces membres ne sont pas satisfaits, en tout ou en partie, du programme adopté(31). Le ministre du Travail bénéficie d'un pouvoir discrétionnaire d'accepter ou non de désigner un arbitre: c'est d'ailleurs le ministre qui transmet au comité et à l'arbitre la liste des points de désaccord, en retenant ou écartant ceux mentionnés dans la demande d'arbitrage. L'art. 224 (3) précise au demeurant que cette liste doit se limiter aux points «que le ministre

(25) Tel est le cas en matière de télécommunications, de transport inter-provincial, de navigation fluviale, de transport aérien, d'énergie atomique, de banques, etc.

(26) L.R.C. (1985), c. L-2., art. 211 et s., C. c. t.

(27) Art. 212 (1), C. c. t.

(28) Art. 213. (1) C. c. t.

(29) Art. 214 C. c. t.

(30) Art. 221, C. c. t.

(31) Art. 233 (1) C. c. t. 
estime pertinents et qui sont normalement du ressort des conventions collectives » (32).

L'arbitre a pour mission d'aider le comité mixte à élaborer un programme d'adaptation et, le cas échéant, de décider des points de désaccord figurant sur la liste établie par le ministre. Toutefois, l'arbitre ne peut ni réviser la décision de l'employeur de procéder à des licenciements, ni retarder son exécution(33). La décision de l'arbitre est finale et sans appel (art. 56 et 225 C. c. t.). Au contraire du droit québécois du travail, le Code canadien du travail ne prévoit pas le versement d'une indemnité aux surnuméraires si l'employeur fait défaut de produire l'avis requis. Toutefois le salarié concerné a droit à un préavis de départ et, s'il a été à l'emploi de l'employeur sans interruption pendant au moins un an, à une modeste indemnité de départ(34).

\section{L'autonomie collective}

L'article 229 C. c. t. prévoit que les dispositions relatives aux licenciements collectifs ne s'appliquent pas si la convention collective prévoit des mécanismes de négociation et de règlement définitif en cas de licenciements, ou des mesures visant à en minimiser les conséquences et à aider les licenciés à trouver un autre travail. Le Code canadien du travail reconnaît ici le fait qu'en milieu syndiqué, les dispositions des conventions collectives sont généralement plus avantageuses pour les salariés que celles que l'on retrouve à la section III (relatives aux normes minimales du travail) du Code canadien. Il en va de même pour le chapitre IV («Normes du travail») de la loi sur les normes du travail du Québec. On doit toujours être attentif au fait qu'en contexte québécois une large part du droit applicable aux milieux syndiqués provient des accords collectifs de travail et non du droit étatique.

L'impact des conventions collectives en matière de licenciements et de fermetures d'entreprise se manifeste à divers niveaux (HÉBERT et alii, 2003): l'établissement d'un plancher d'emploi ou d'une garantie d'emploi, ce qui existe uniquement dans le secteur public et parapublic; l'avis de mise à pied, dont la durée sera plus longue que celle prévue par la loi ou qui donnera lieu à une indemnité compensatrice; les modalités de mise à pied, lesquelles reposent généralement sur le respect du principe de l'ancienneté; la procédure de rappel au travail, qui limite la marge de manœuvre de l'employeur en matière de licenciements; enfin, les indemnités de fin d'emploi, lesquelles représentent parfois des montants substan-

(32) Voir infra, la section consacrée à «l'autonomie collective».

(33) Art. 224 (5), C. c. t.

(34) Voir les art. 230, 212 (1) et 235 (1) C. c. t. tiels (35). L'effet des conventions collectives sur les licenciements collectifs au Québec n'a pas été étudié de manière systématique; par ailleurs, des études de cas sont nécessaires pour mesurer l'effectivité du droit issu de l'autonomie collective comme mesure palliative aux licenciements et fermetures. De telles études empiriques restent encore à réaliser. Ce qui demeure certain toutefois, c'est que le droit canadien et québécois du travail, étatique ou non, ne permet pas vraiment de remettre en question la décision de l'employeur de procéder à des licenciements collectifs ou à des fermetures d'établissement. Les mesures d'accompagnement prévues par le droit étatique sont minimales et peu contraignantes, et les indemnités de licenciement extrêmement faibles. Les conventions collectives contiennent généralement des normes plus favorables (encore que le versement d'indemnités substantielles de licenciement soit exceptionnel).

Licenciements collectifs et fermetures relèvent, en contexte québécois et canadien, d'une conception quasi absolutiste des droits de direction. Le cas WalMart est tout à fait révélateur de cet état des choses: nous nous trouvons ici face à un cas-limite, celui où une entreprise utilise l'arme des restructurations (36), non pour des raisons purement économiques, mais pour des motifs en partie idéologiques(37) qui tiennent à son antisyndicalisme viscéral (38). Ce caslimite, qui normalement devrait être réprouvé sévèrement par la loi, illustre éloquemment la faiblesse du droit québécois du travail face à de telles circonstances, à tout le moins suivant l'interprétation dominante qui en est faite. Il ne faut pas, par ailleurs, banaliser le phénomène: à la faveur de la libéralisation des échanges à l'échelle nord-américaine, de nombreuses entreprises transnationales en provenance des ÉtatsUnis investissent massivement le secteur des services (alimentation, restauration, vente au détail, marché de la rénovation, etc.) au Canada et au Québec, en se montrant elles aussi réfractaires à la syndicalisation. Il est question ici d'emplois peu spécialisés et faiblement rémunérés, dans un secteur qui génère pourtant des revenus considérables; l'effet sur les entreprises concurrentes, canadiennes et québécoises, établies dans ce secteur est délétère, puisqu'il y a partout tendance à rogner sur les «privilèges» accordés aux travailleurs syndiqués ou même à tenter de résister à la concurrence en ouvrant des succursales non syndiquées.

(35) Par exemple, lors de la fermeture de l'usine GM de Boisbriand, les salariés de 50 ans et plus ont reçu une indemnité de départ de 40 à $50000 \$$. Il s'agit là, toutefois, d'un cas tout à fait exceptionnel.

(36) Sur le sens de cette notion, voir RaveYre (2005/1) p. 10.

(37) En grande partie économiques aussi, bien sûr, puisque Wal-Mart rémunère ses salariés jusqu'à $40 \%$ moins que ses concurrents. V. The Economist, 26 février 2005, p. 37.

(38) Voir Fortune, le 18 avril 2005, v. 151, p. 84 et s. : When it comes to unions, Wal-Mart's stance is implacable. And this fight may provide the clearest example yet of how the company's adherence to the old ways of doing business is hurting it in the present. 


\section{Un cas limite: Wal-Mart ou les restructurations comme stratégie antisyndicale}

Wal-Mart représente la plus grande entreprise de détail au monde et le plus grand employeur privé, avec plus de 1700000 salariés. En 2004, le chiffre d'affaires de l'entreprise était de 288 milliards \$(39). Établie au Québec depuis 1994, Wal-Mart y exploite présentement quarante-sept magasins et emploie 10000 salariés qu'elle qualifie, sans ironie, «d'associés» (40). Jusqu'à récemment, aucun établissement de cette énorme transnationale n'était syndiqué en Amérique du Nord. En fait, le premier établissement Wal-Mart qui a fait l'objet d'une accréditation syndicale fut celui de Jonquière au Québec (région du Lac Saint-Jean), en août 2004. Ce même établissement fut fermé par l'entreprise le 6 mai 2005, prétendument parce que non rentable(41).

Le 11 mai 2005 (42), la Commission des relations du travail du Québec (CRT) a rendu une première décision importante dans le cas du Wal-Mart de Jonquière. On ne peut que regretter le manque d'audace, le conservatisme social et le caractère expéditif de cette décision. Il s'agissait en l'occurrence d'une demande d'ordonnance provisoire de sauvegarde (43), présentée par les travailleurs et travailleuses unis de l'alimentation et du commerce (TUAC) à l'encontre de Wal-Mart Canada inc., et ayant pour objet la fermeture du magasin de Jonquière. Les TUAC demandaient à Wal-Mart de reprendre les activités normales de ce magasin et de les maintenir jusqu'à ce qu'intervienne une décision au fond dans cette affaire; l'employeur était en effet poursuivi devant la CRT pour son comportement antisyndical. Or, l'argumentation des TUAC fut rejetée du revers de la main, bien que le sérieux des allégations et leur portée juridique et sociale éminente auraient mérité un examen beaucoup plus attentif et rigoureux. Deux aspects doivent être soulignés: la liberté fondamentale d'association des salariés; le droit à la fermeture de l'entreprise.

\section{La liberté fondamentale d'association}

Les représentants des TUAC ont soulevé des arguments constitutionnels, mais ceux-ci n'ont pas

(39) Fortune, le 18 avril 2005, v. 151, p. 84.

(40) À l'échelle du Canada, Wal-Mart compte 262 succursales et plus de 60000 salariés.

(41) Les salariés d'un second établissement Wal-Mart au Québec, celui de Saint-Hyacinthe, ont obtenu une accréditation syndicale, mais aucune convention collective n'a encore été signée avec l'employeur.

(42) Boutin et Travailleurs et travailleuses unis de l'alimentation et du commerce c. Wal-Mart Canada inc., 2005 QCCRT 0269, le 11 mai 2005.

(43) En matière administrative, équivalent de l'injonction laquelle est du ressort exclusif des cours supérieures de justice. Voir l'art. 127 C. t. été pris en considération par la CRT dans sa décision du 11 mai 2005. Les prétentions du syndicat sont résumées en un court paragraphe(44) qui ne fait aucune référence explicite à cette argumentation. Pourtant, la valeur prééminente des libertés et droits fondamentaux pour la sphère des rapports de travail a été rappelée avec force par la Cour suprême du Canada dans des décisions récentes (45).

Certes, la Cour suprême du Canada a, depuis sa désolante «trilogie» de 1987(46), estimé que la liberté d'association ne devait pas s'évaluer en fonction des «prétendues exigences des syndicats» (47) et que le libre choix de l'unité d'accréditation n'était pas garanti par la charte canadienne des droits et libertés (article 2d)(48), puisque l'octroi de l'accréditation fait partie du processus de négociation, lequel ne bénéficie d'aucune protection constitutionnelle au Canada. Adopter cette interprétation serait toutefois méconnaître le récent délaissement par la Cour suprême du strict libéralisme économique qui caractérisa pendant plusieurs années sa position, au profit d'une lecture davantage sociale de la liberté d'association - encore que bien insuffisante si on la juge à l'aune du droit international du travail(49). Dans la décision Dunmore c. Ontario (Procureur général)(50), la Cour suprême a reconnu pour la première fois la dimension collective, et non plus seulement individuelle, de la liberté constitutionnelle d'association. Qui plus est, la Cour, se référant aux conventions internationales du travail, s'est montrée d'avis que le déni par l'État d'une reconnaissance officielle (par voie d'accréditation ou autrement) des associations syndicales des travailleurs agricoles était incompatible avec la garantie constitutionnelle de la liberté d'association. L'extrait suivant résume bien la position de la Cour suprême: «La liberté syndicale est aussi difficile à exercer qu'elle est fondamentale, et qu'elle forme une pièce unique dans la toile constitutionnelle canadienne où l'histoire a tissé une

(44) Décision précitée, paragr. 33

(45) Parry Sound (District), conseil d'administration des services sociaux c. SEEFPO, section locale 324, [2003] 2 RCS 157 ; Québec (Commission des droits de la personne et des droits de la jeunesse) c. Québec (procureur général), [2004] 2 RCS 27.

(46) Il s'agit ici de trois arrêts rendus le 9 avril 1987 et où la majorité de la Cour se refuse à donner la moindre protection constitutionnelle, sous l'angle de la liberté d'association, à la liberté syndicale.

(47) Re Public Service Employees Relations Act, [1987] 1 R.C.S. 313, p. 390 (motifs du juge Le Dain).

(48) La carte canadienne des droits et libertés fait partie de la Constitution du Canada (loi constitutionnelle de 1982, L.R.C. 1985, App. II, no 44).

(49) Pour une critique des positions de la Cour suprême, voir Brunelle, Verge (2003).

(50) [2004] 1 R.C.S. 43. Le litige portait sur la validité constitutionnelle de la politique du gouvernement de l'Ontario se refusant à reconnaître la liberté syndicale des travailleurs agricoles, lesquels demeuraient exclus de la loi sur les relations de travail. 
protection légale»(51). En l'occurrence, la Cour tient compte de la situation de faiblesse qui caractérise historiquement la position des travailleurs agricoles, leur manque de ressources et leur grande vulnérabilité face aux représailles patronales.

L'arrêt Dunmore se transpose aisément au contexte de l'article 3 de la charte des droits et libertés de la personne du Québec(52), qui est le texte de nature («quasi») constitutionnelle qui s'applique ici, vu que les TUAC tout comme WalMart Canada inc. sont évidemment des entités non gouvernementales (donc, non visées par la charte canadienne des droits et libertés)(53). L'article 3 de la charte québécoise garantit à toute personne un certain nombre de libertés classiques, dont la liberté d'association. Porte atteinte à la liberté d'association, croyons-nous, la décision d'un employeur, motivée par un animus antisyndical, de fermer un de ses magasins, cette fermeture visant (ce qui nous paraît évident, comme à la plupart des observateurs, dans le cas de Wal-Mart inc.) à faire échec à une tentative de syndicalisation. En l'occurrence, le sort des travailleurs du commerce de détail, faiblement rémunérés, vulnérables aux pressions patronales, peu organisés et à la merci de puissantes entreprises multinationales souvent (comme c'est le cas de WalMart inc.) farouchement antisyndicales, est à peine plus enviable que celui des travailleurs agricoles.

Pourtant, le seul droit qualifié de «fondamental» dans la décision du commissaire - lequel ne prête aucune attention à la liberté constitutionnelle d'association - est «le droit fondamental de fermer son entreprise». Celui-ci constitue évidemment un attribut du droit de propriété, lequel ne bénéficie pourtant que d'une protection relative («sauf dans la mesure prévue par la loi») en vertu de l'article 6 de la charte des droits et libertés de la personne du Québec. L'exercice du droit de propriété peut-il être considéré - car c'est bien en ces termes qu'il faut poser le problème - comme une limitation du droit constitutionnel à la liberté d'association qui se justifie, dans les circonstances, dans le cadre d'une société libre et démocratique?(54) Ceci nous conduit à étudier de plus près le «droit de fermer son entreprise» en tant qu'attribut du droit de propriété.

(51) Décision précitée, paragr. 35.

(52) L.R.Q., c. C-12. La charte québécoise a, suivant la Cour suprême du Canada, valeur «quasi constitutionnelle». Elle fait partie de la Constitution du Québec en un sens matériel.

(53) La charte québécoise s'applique tant à l'activité privée qu'aux actes de l'État; la charte canadienne ne concerne pour sa part que la sphère étatique (entendue largement toutefois). (54) La Cour suprême du Canada applique un test de rationalité et de proportionnalité pour évaluer si, conformément à l'article premier de la charte canadienne des droits et libertés, la limitation d'une liberté fondamentale se justifie dans le cadre d'une société libre et démocratique. Le même test prévaut en ce qui concerne les restrictions aux libertés fondamentales garanties par la charte québécoise (art. 9.1.).

\section{Le droit de l'employeur à la fermeture de son entreprise}

Le commissaire de la CRT fait référence, sans inscrire aucunement sa réflexion dans le cadre d'une analyse fondée sur les libertés et droits de la personne, à la décision de la Cour suprême du Canada dans Société de la Place des Arts de Montréal(55), qui traite incidemment de la question de la fermeture d'une entreprise. Dans cette décision, la Cour suprême a jugé que la Société de la Place des Arts ne contrevenait pas aux dispositions antibriseurs de grève du Code du travail en cessant de fournir aux locataires de la Place des Arts les services techniques autrefois assumés par le syndicat appelant (qui avait entrepris une grève légale) et maintenant placés sous la responsabilité directe des locataires (tels l'Orchestre symphonique et les Grands ballets canadiens). Le vice-président de la CRT cite certains extraits de cette décision de la Cour suprême du Canada, laquelle se réfère au passage suivant d'une décision antérieure du tribunal du travail (juge Lesage): «Si un employeur, pour quelle que raison que ce soit, décide par conséquent de véritablement fermer boutique, les congédiements auxquels il procède sont causés par la cessation de ses activités, ce qui est une raison économique valable de ne pas engager de personnel, même si cette cessation est mue par des motifs condamnables socialement» (56).

Certes, la CRT mentionne que cette fermeture doit cependant être faite «de bonne foi», mais elle s'avoue incapable de conclure à un manquement à cette exigence, aucun élément de preuve n'ayant été fourni, semble-t-il, pour démontrer une intention de Wal-Mart de continuer à opérer les activités du magasin de Jonquière «indirectement, avec l'aide d'autrui, ailleurs ou d'autres façons ou encore après l'écoulement d'un laps de temps pour sauver les apparences». Il y a ici une conception pour le moins étriquée de la notion «d'entreprise» lorsqu'il est question d'une multinationale de la puissance de Wal-Mart(57). Mais à notre avis il y a aussi une compréhension bien insatisfaisante de la décision de la Cour suprême dans l'affaire Place des Arts. Cette décision est plus nuancée que la CRT ne se la représente, et surtout doit être interprétée en tenant compte des circonstances propres au litige.

a) Dans Place des Arts, la Société et ses locataires constituaient des entités juridiques distinctes, «supportant les risques économiques et en tirant

(55) Société de la Place des Arts de Montréal c. Alliance internationale des employés de scène, de théâtre, du cinéma, des métiers connexes et des artistes des États-Unis et du Canada, local de scène numéro 56, [2004] 1 R.C.S. 43.

(56) Citiy Buick Pontiac (Monréal) inc. c. Roy, [1981] T.T. 22.

(57) Voir à ce sujet l'article de Jean-Guy Belley et Patrick Forget de l'université McGill dans Le Devoir du 24 mai 2005, p. 7. 
des bénéfices de manière individuelle» (58), ce qui n'est évidemment pas le cas lorsque l'on considère les 262 magasins qui demeurent la propriété directe de Wal-Mart Canada inc. Une seule et même entité juridique est ici présente.

b) Dans Place des Arts, la Cour suprême reconnaît le droit d'une entreprise de cesser d'opérer partiellement ses activités: mais l'activité faisant l'objet d'une cessation d'opération est bien délimitée, il s'agit des services techniques fournis aux locataires. Ce type d'activité spécifique ou «entreprise» est totalement abandonnée par la Société de la Place Des Arts: rien de tel chez Wal-Mart qui ne délaisse évidemment pas son activité de commerce de détail, même sur une base régionale, car elle opère simultanément, à quelques kilomètres de distance, des points de vente à Chicoutimi et Alma.

c) Tout en faisant sien l'énoncé du juge Lesage voulant que l'employeur puisse fermer son entreprise «même pour des motifs socialement condamnables», la Cour suprême souligne que «les tribunaux judiciaires et administratifs doivent examiner attentivement les faits propres à chaque espèce pour s'assurer que la décision de fermer l'entreprise est réelle et non pas simplement un subterfuge...»(59). Le juge Lesage ajoutait lui-même, énoncé repris par la Cour suprême, que «c'est certes avec la plus grande sévérité qu'il faut scruter les circonstances de la décision de fermeture pour en apprécier le véritable caractère» (VERGE, Roux, 2006, pp. 223 263).

Peut-on croire que la CRT ait procédé ici à un examen attentif des faits et en ait scruté les circonstances «avec la plus grande sévérité»? Le caractère lapidaire des motifs en laisse grandement douter. Même s'il s'agit assurément de notions floues, le droit québécois du travail reconnaît depuis longtemps la distinction entre l'entreprise (la ou les finalités poursuivies par l'employeur) et l'établissement (le lieu physique) : on devrait reconnaître ici que s'il y a fermeture par Wal-Mart Canada inc. de l'un de ses établissements, il n'y a en aucune façon cessation d'entreprise, en tout ou en partie (60).

À tout événement, pour en revenir à la liberté fondamentale d'association garantie par les chartes, on ne peut nullement avancer, à notre avis, que la fermeture d'un établissement, quoique sous-tendue par le droit de propriété, constitue dans ces circonstances une mesure qui se justifie dans le cadre d'une société libre et démocratique. Une telle mesure ne rencontre aucunement les critères de rationalité et de proportionnalité de l'article 9.1 de la charte

(58) Arrêt Place des Arts, paragr. 20.

(59) Idem, paragr. 29.

(60) Voir la position au même effet de Guylaine Vallée, Gilles Trudeau et Gregor Murray dans Le Devoir, édition du 15 février 2005. québécoise lorsqu'elle a pour seul objectif de brimer l'exercice de la liberté fondamentale d'association d'un groupe de salariés en situation de faiblesse et de vulnérabilité (61).

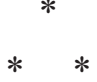

Le nombre de licenciements collectifs et de fermetures d'usines et d'établissements a augmenté de manière dramatique au Québec. Il ne se passe pas une semaine sans que l'annonce de telles mesures ne soit diffusée par les médias. Le niveau de chômage se maintient toutefois à environ $9 \%$ (ce qui n'est pas du tout un niveau record pour le Québec), la création de nouveaux emplois compensant au total l'impact négatif des restructurations, (un agent du ministère du Travail du Québec, 2005). Par ailleurs, c'est le secteur des services (notamment la gestion, l'assurance et l'immobilier, l'information et les loisirs) qui absorbe la quasi-totalité des gains d'emploi, alors que le secteur de la production est en relatif déclin (idem). Enfin, le travail précaire et atypique occupe une part croissante dans la répartition globale de l'emploi, allant jusqu'à représenter $25 \%$ de la main-d'œuvre (BerNier, ValléE, JoBin, 2003).

Ces tendances de fond traduisent l'adaptation du marché québécois de l'emploi à la «nouvelle économie» globalisée. La flexibilité accrue de la main-d'œuvre signifie, du point de vue des travailleurs, l'insécurité, une faible rémunération et une augmentation globale du niveau de pauvreté (ibidem,p. 40). Le développement du travail précaire, ainsi que le déplacement de l'emploi du secteur de la production vers celui des services, fragilise les acquis du droit social, puisque les secteurs et formes d'emploi en croissance sont faiblement syndiqués et difficiles à organiser. Il y a là un effet d'exclusion de la démocratie industrielle et de la citoyenneté au travail qui mine à son tour les fondements de la démocratie sociale et de l'État social.

(61) Une requête en révision de cette décision présentée par le TUAC a été rejetée par trois commissaires de la CRT en juillet 2005. Voir Boutin c. Wal-Mart Canada inc., 2005 QCCRT 385, le 7 juillet 2005. Dans cette décision, la CRT laisse ouverte la question d'une distinction entre l'établissement et l'entreprise, estimant que cet argument aurait dû être soulevé à la première occasion. Par ailleurs, dans Bourgeois c. Compagnie Wal-Mart du Canada (C.R.T., 2005-09-15), 2005 QCCRT 0502, SOQUIJ AZ-50333563, D.T.E. 2005T-840, la CRT a fait droit à trois plaintes (sur 4) de congédiements pour activités syndicales, suite à la fermeture du Wal-Mart de Jonquière. La seule raison pour laquelle ces plaintes sont accueillies tient à l'absence de preuve convaincante d'une fermeture définitive de l'établissement de Jonquière, Wal-Mart détenant toujours le bail locatif de ce magasin. Cette décision ne reconsidère aucunement la position de la CRT en matière de fermeture d'entreprise et demeure muette quant aux arguments constitutionnels soulevés par les TUAC. 
Les lourds silences du droit face à l'épidémie de fermetures d'usines et de licenciements collectifs contribuent directement au déclin de la citoyenneté au travail (COUTU, MURRAY, à paraître). La perte de dizaine de milliers d'emplois syndiqués ne peut se traduire à terme que par une baisse du niveau de syndicalisation et un affaiblissement correspondant du syndicalisme québécois, lequel joue un rôle essentiel de contrepoids à l'individualisme néolibéral et contribue de manière décisive à la qualité de la vie démocratique.

S'agit-il ici d'une sorte de fatalité, faut-il constater l'impuissance du mouvement syndical ou de l'État social face à la dynamique aveugle de la mondialisation?

Rien n'excuse à notre avis les silences du droit. Même dans un contexte globalement défavorable au droit social, le National Labor Relations Board américain (NLRB), sur la base des dispositions très générales du National Labor Relations Act (op. cit. supra)-nullementplusprécisesquecelles, similaires, que contient le Code du travail du Québec - a jugé illégales les fermetures d'usines motivées par un animus antisyndical(62). Les employeurs violant la loi ont été condamnés à verser des dommagesintérêts et le NLRB a même, à plusieurs reprises, ordonné la réouverture de l'usine ou de l'entreprise. On est loin ici, tout étonnant qu'il puisse paraître car nous parlons des États-Unis, chef-lieu de l'individualisme libéral, du seul «droit fondamental de tout employeur» à fermer un établissement «même pour des motifs antisociaux » qu'a brandi à la légère la CRT québécoise face à la hargne anti-syndicale de Wal-Mart. Les dispositions du Code du travail du Québec protégeant la liberté syndicale et l'exercice du droit d'association nous semblent suffisantes, dans leur libellé actuel, pour disposer de ce genre de situations où un employeur vise à contrecarrer la syndicalisation de ses travailleurs ou à se débarrasser du syndicat (63).

Toutefois, dans la grande majorité des cas, la fermeture d'une usine ou d'un établissement, ou bien des licenciements collectifs, répondent à une

(62) Voir (réouverture de l'établissement ordonné) Dorsey Trailers, Inc. Northumberland, PA Plant v. United Auto Workers International Union and its Local 1868, International Union, AFL-CIO, CLC. NLRB Cases 4-CA-23996, 4-CA-24049, 4-CA-24356, and 4-CA-24423 (March 12, 1999), infirmé toutefois par Dorsey Trailers, Inc. Northumberland, PA Plant v. NLRB, 233 F. 3d at 839 (C.A., $4^{\text {th }}$ Cir., 2000). V. aussi (octroi de dommages-intérêts) : Contech Division, SPX Corp. v. UAW, 320 NLRB no 52 (1995). NLRB v. Champion Laboratories Inc., C.A. 7th. Cir., no 95=2433 (1996). Cable-Masters inc. v. Communications Workers of America, 307 NLRB no 139 (1992). Amercia's Best Qualitiy Coatings Corp. and Staff Right inc. v. United Electrical Workers, 313 NLRB no 52 (1993). General Electric Company v. United Electrical, Radio and Machine Workers of America, 321 NLRB no 86 (1996).

(63) Voir en ce sens : Banque nationale du Canada c. Union internationale des employés de commerce, [1984] 1 R.C.S. 269. motivation d'ordre purement économique, celle de la rentabilité et du profit. Lorsqu'il est question uniquement de la rationalité propre au système économique, entre-t-on pour autant dans une sphère de non-droit?

Le Code du travail du Québec contient, à l'instar de toutes les lois visant les rapports collectifs de travail en Amérique du Nord, une disposition tout à fait centrale qui oblige les parties à négocier de bonne foi (64). Suivant la Cour suprême du Canada, le devoir de négocier (duty to bargain) comprend deux aspects: l'un, subjectif, qui concerne l'attitude des parties à la table de négociation; l'autre, objectif, qui oblige à faire «tout effort raisonnable pour conclure une convention»(65) et s'apprécie suivant les normes et pratiques comparables dans le secteur d'activité en cause(66). En Ontario, le devoir de négocier a été mis en application par la commission des relations de travail dans le contexte d'une fermeture d'usine: l'annonce de celle-ci survenant peu après la signature de la convention collective, sans avoir jamais été invoquée par l'employeur, fut considérée comme un manquement au devoir de négocier (67). Au Canada et au Québec, il nous semble que le devoir de négocier de bonne foi a été, à date, invoqué exclusivement dans le cadre du délai formel de négociation (68) ou par rapport à des manquements survenus durant cette période. Par contre, aux États-Unis - où les dispositions sont en gros similaires - le fait pour l'employeur de prendre une décision unilatérale affectant les «conditions de travail» de ses salariés, sans négociation préalable, représente un manquement au devoir de négocier de bonne foi(69). Toutefois, tel que mentionné, par «conditions de travail» ne sont visées ici que les matières «obligatoires» de négociation et non les sujets facultatifs. Considérés comme relevant de cette dernière catégorie, les décisions relatives aux fermetures d'usine et aux licenciements collectifs ne donnent pas ouverture à une plainte de manquement au devoir de négocier

(64) Voir l'article 53, second alinéa, du Code du travail du Québec (L.R.Q., c. C-27) : «Les négociations doivent commencer et se poursuivre avec diligence et bonne foi».

(65) Royal Oak Mines Inc. c. Canada (C.R.T.), [1996] 1 R.C.S. 369 , p. 397.

(66) Le Code canadien du travail, art. 50, alinéas a) (i) et (ii) est beaucoup plus précis à cet égard que l'article $53 \mathrm{C}$. $\mathrm{t}$. Q. La doctrine estime néanmoins que les différences terminologiques ne font pas obstacle à l'application de la jurisprudence Royal Oak Mines au Québec. V. Robert P. Gagnon (2003, p. 410 et s).

(67) International Woodworkers of America, Local 2-69 v. Consolidated Bathurst Packaging Ltd., [1983] O.L.R.B. Rep. 1411.

(68) Le droit nord-américain du travail n'autorise la négociation collective qu'à certaines périodes déterminées, liées à la date d'expiration de la convention collective. Voir ainsi les articles 52 et s. du C. t.

(69) First National Maintenance Corp. v. NLRB, 452 U.S. 666 (1981). 
de bonne foi. Par contre, les effets de telles décisions doivent faire l'objet d'une négociation.

Compte tenu de la similarité des dispositions en cause, nous croyons que le devoir de négociation de bonne foi devrait être compris - que l'on soit ou non en période formelle de négociation-comme imposant à l'employeur l'obligation de négocier avec le syndicat lorsqu'il envisage des fermetures d'usines ou des licenciements collectifs. Cette négociation ne devrait pas porter uniquement sur les effets ( $i$. e. le reclassement, les indemnités de licenciements, la mise à la retraite, etc.) de la mesure sur les travailleurs concernés, comme c'est le cas aux États-Unis, mais aussi sur ses fondements. Le droit canadien récuse en effet, tel que mentionné, la définition états-unienne des conditions de travail comme ne visant que les questions «obligatoires» : toute question, soulevée par l'une ou l'autre des parties et pouvant faire l'objet d'une négociation collective, est considérée comme étant une «condition de travail »(70).
Au-delà des dispositions actuelles, bien insuffisamment utilisées à notre avis, du droit québécois du travail, l'intervention de l'État apparaît certes indispensable. Le droit européen des licenciements collectifs est susceptible de fournir ici un modèle de référence, tout en exigeant évidemment une transposition tenant compte du contexte spécifique des relations industrielles au Québec. Deux impératifs nous semblent s'imposer, du point de vue d'une révision éventuelle de la législation du travail: l'obligation d'une négociation préalable des fondements mêmes de la décision patronale, pour voir si des solutions moins draconiennes ne sont pas possibles dans les circonstances et ce, tant chez les non syndiqués qu'en milieu syndiqué (71); le cas échéant, la nécessité (qui impliquerait un allongement très significatif de la période de préavis) d'une négociation des effets de la restructuration envisagée sur la main-d'œuvre concernée, pour en limiter autant que possible l'impact négatif.
(70) Syndicat catholique des employés de magasin de Québec c. Cie Paquet Ltée, [1959] R.C.S. 206.

(71) À notre avis, ceci pourrait être l'occasion d'une mise en cohérence de la législation du travail, qui exige actuellement la formation de plusieurs comités paritaires, même en milieu non syndiqué : on retrouve ainsi des comités paritaires en matière de santé et de sécurité du travail, en matière d'équité salariale ou en cas de licenciements collectifs. Il y aurait lieu ici de

\footnotetext{
s'inspirer du modèle européen pour imposer, dans tout lieu de travail, la formation d'un comité d'entreprise, lequel assumerait ces diverses responsabilités et d'autres encore que le législateur voudrait bien lui confier. Bien entendu, en milieu de travail syndiqué, il faudrait s'assurer d'une articulation étroite entre le syndicat accrédité et les délégués représentant les travailleurs au sein du comité d'entreprise (nomination des délégués par l'instance syndicale).
} 


\section{Bibliographie}

Adams R.J. (1995), Industrial Relations under Liberal Democracy: North America in Comparative Perspective, Columbia, University of South California Press.

Arthurs H. (1996), "Labour Law without the State?" 46 University of Toronto Law Journal 1-45.

BéliveauN.-A (2003), Lesnormes dutravail, Cowansville (Québec), Éditions Yvon Blais, p. 252.

Bernier J., Vallée G., Jobin C. (2003), Les besoins de protection sociale des personnes en situation de travail non traditionnelle, rapport du Comité d'étude, Québec, ministère du Travail, p. 38.

Brunelle Ch., Verge P. (2003), «L'inclusion de la liberté syndicale dans la liberté générale d'association: un pari constitutionnel perdu?», 82 R. du B. Can. 711-755.

Clarkson S. (2002), Uncle Sam and Us. Globalization, Neoconservatism and the Canadian State, University of Toronto Press, p. 310.

Coutu M., Murray G. (sous la direction de) (2007), Travail et citoyenneté: Quel Avenir? Québec, Les Presses de l'université Laval, (à paraître).

Gagnon R.-P. (2003), Le droit du travail au Québec, 5 édition, Cowansville (Qué.), Éditions Yvon Blais, p. 410 et $\mathrm{s}$.

Gurvitch G. (1931), Le temps présent et l'idée de droit social, Paris, Sirey.

Habermas J. (1981), Raison et légitimité. Problèmes de légitimation dans le capitalisme avancé, Paris, Payot.

HéBert G. et alii, (2003), La convention collective au Québec, Boucherville (Québec), Gaëtan Morin éditeur, 2003, pp. 193 et s.

Johnson S. (2004), “The Impact of Mandatory Votes on the Canada-US Union Density Gap: A Note", 43 Industrial Relations 356.

LipSET S.M. (1990), Continental Divide: the Values and Institutions of the United States and Canada, New York, Routledge.

Merrien F.-X., Parchet R., Kernen A. (2003)., L'État social. Une perspective internationale, Paris, Dalloz/ Armand Colin, pp. 262 et s.
Offe C. (1984), Contradictions of the Welfare State, Cambridge, MIT Press.

RAVEYRE M. (2005/1), «Introduction», dans Restructurations. Nouveaux enjeux, La Revue de l'IRES, $\mathrm{n}^{\mathrm{0}} 47,7-17$, p. 10.

Rice J.J., Prince M.J. (2000), Changing Politics of Canadian Social Policy, University of Toronto Press, chapter 4: "The Crisis of the Welfare State: Canadian Perspectives and Critiques".

Rosanvallon P. (1984), La crise de l'État-providence, Paris, Le Seuil, 1981.

Rose J.B., Chaison G.N. (1996), "Linking Union Density and Union Effectiveness: The North American Experience", 35 Industrial Relations 78.

Rose J.B., Chaison G.N. (2001), "Unionism in Canada and the United States in the 21th Century. The Prospects for Revival", 56 Industrial Relations 34.

SchnAPPer D. (2002), La démocratie providentielle. Essai sur l'égalité contemporaine, Paris, Gallimard.

Sinzheimer H. (1976), 'Die Demokratisierung des Arbeitsverhältnisses (1928)', in Arbeitsrecht und Rechtssoziologie. Gesammelte Aufsätze und Reden, tome 1, Francfort, Europaïsche Verlagsanstallt, pp. 108-114.

STRÅth Bo (2003), “The State and its Critics: Is There a Post-Modern Challenge?", in Q. SkInNER, Bo StrÅth, States \& Citizens. History, Theory, Prospects, Cambridge University Press, pp. 167-190.

Verge P., Roux D. (2006), «Fermer l'entreprise: un droit absolu?», in Développements récents en droit du travail, Service de la formation continue du barreau du Québec 2006 no 245, Cowansville (Qué.), Éditions Yvon Blais, pp. 223-263.

Weiler P. (1983), "Promises to Keep: Securing Workers'Rights to Self-Organization under the NLRA", Harvard L. R. 1769.

ZACHERT U. (2006), «La légitimité des rapports juridiques de travail. À propos de la conception de la légitimité chez Max Weber et Hugo Sinzheimer», in M. Coutu, G. Rocher (sous la direction de), La légitimité de l'État et du droit. Autour de Max Weber, Québec/Paris, Les Presses de l'université Laval/LGDJ, pp. 301-331. 